\title{
Subliminal priming with nearly perfect performance in the prime-classification task
}

\author{
Matthew Finkbeiner
}

Published online: 29 January 2011

(C) Psychonomic Society, Inc. 2011

\begin{abstract}
The subliminal priming paradigm is widely used by cognitive scientists, and claims of subliminal perception are common nowadays. Nevertheless, there are still those who remain skeptical. In a recent critique of subliminal priming, Pratte and Rouder (Attention, Perception, \& Psychophysics, $71,1276-1283,2009)$ suggested that previous claims of subliminal priming may have been due to a failure to control the task difficulty between the experiment proper and the prime-classification task. Essentially, because the primeclassification task is more difficult than the experiment proper, the prime-classification task may underestimate the subjects' true ability to perceive the prime stimuli. To address this possibility, prime words were here presented in color. In the experiment proper, priming was observed. In the prime-classification task, subjects reported the color of the primes very accurately, indicating almost perfect control of task difficulty, but they could not identify the primes. Thus, the present findings suggest that controlling for task difficulty does not eliminate subliminal priming.
\end{abstract}

Keywords Subliminal priming - Visual awareness .

Prime identification

\section{Introduction}

The masked priming paradigm is widely used in the investigation of word and object recognition (Forster \& Davis, 1984; Grainger, Colé, \& Segui, 1991), as well as in

M. Finkbeiner $(\bowtie)$

Macquarie Centre for Cognitive Science, Macquarie University, Sydney, NSW 2109, Australia

e-mail: matthew.finkbeiner@mq.edu.au the investigation of nonconscious processes (Abrams, Klinger, \& Greenwald, 2002; de Gardelle \& Kouider, 2010; Dehaene et al., 1998; Finkbeiner \& Palermo, 2009; Pessiglione et al., 2007). In a standard version of the subliminal priming paradigm, subjects are asked to categorize a target stimulus (e.g., the word TIGER) into one of two groups (e.g., "Is it an animal or a vegetable?"). Importantly, in this paradigm the target stimulus is preceded by a prime stimulus (e.g., the word "dog") that is related to the target in one way or another. The dependent measure is usually response times (RTs) in the case of behavioral paradigms, but the paradigm has also been used by cognitive neuroscientists with a wide range of neurophysiological measures, including eventrelated potentials (Kiefer \& Brendel, 2006), lateralized readiness potentials (Eimer \& Schlaghecken, 1998), motor evoked potentials (Verleger, Kötter, Jaśkowski, Sprenger, \& Siebner, 2006), and BOLD signal changes in fMRI studies (Dehaene et al., 1998; Kouider, Eger, Dolan, \& Henson, 2009; Pessiglione et al., 2007). A subliminal priming effect is obtained when undetectable prime stimuli modulate targetrelated processes in some way. Take, for example, an experiment in which subjects have to classify targets as animals or vegetables. If the congruent prime-target pairs dog-TIGER and pea-CARROT yield faster responses than the incongruent prime-target pairs pea-TIGER and dog-CARROT, then priming is said to have occurred. If, in a separate task that assesses prime awareness, subjects are not able to identify the prime stimuli at better than chance levels (i.e., $d^{\prime}$ is not statistically different from zero), then subliminal priming is said to have occurred.

In a recent study, Pratte and Rouder (2009) claimed "to provide evidence that previous demonstrations [of subliminal priming] may have been susceptible to a subtle methodological artifact" (p. 1276). Given the widespread use of the masked priming paradigm and the general acceptance 
nowadays that subliminal perception exists (cf. Finkbeiner \& Forster, 2008; Kouider \& Dehaene, 2007, for reviews), Pratte and Rouder's charge is a serious one that carries the potential to undermine a large and active area of research.

Pratte and Rouder (2009) distinguished their critique of masked priming, which focuses on a methodological issue, from the more familiar critiques of subliminal perception that focus on a statistical problem (i.e., the null-sensitivity problem discussed by Reingold \& Merikle, 1988). In the present article, I respond to Pratte and Rouder by first focusing on the methodological artifact that they have investigated, and then I report an experiment designed to address their concerns. Because I observed a subliminal priming effect in the experiment reported below, I will discuss in the analysis section the ways in which I address the more common statistical critique.

In their study, Pratte and Rouder (2009) used the masked congruence priming paradigm and, specifically, the magnitude judgment task ("Is the target less or greater than 5?"). This is perhaps the most commonly used task within the masked congruence priming literature, with numerous studies reporting subliminal priming effects (cf. Dehaene et al., 1998; Koechlin, Naccache, Block, \& Dehaene, 1999; Kouider \& Dehaene, 2009; Kunde, Kiesel, \& Hoffmann, 2003; Reynvoet, Gevers, \& Caessens, 2005; Van den Bussche, Segers, \& Reynvoet, 2008; Van Opstal, Reynvoet, \& Verguts, 2005). As is typical in this literature, Pratte and Rouder had subjects perform two tasks in succession. In the first, they asked their subjects to respond to the visible targets as quickly and accurately as possible, without informing the subjects of the primes. Following Pratte and Rouder, I will refer to this first task as the target-classification task. Then, in the second task, Pratte and Rouder asked subjects to perform a primeclassification task, in which they were instructed to ignore the targets and classify the primes. Pratte and Rouder's main concern with previous reports of subliminal priming stemmed from the way in which most researchers have conducted the prime-classification task. As Pratte and Rouder mentioned, the prime-classification measure is only valid if the primes are equally visible in the target- and prime-classification tasks (see Reingold \& Merikle, 1988). Critically, Pratte and Rouder contended that this assumption is usually violated in subliminal priming experiments.

In most studies, subjects are not informed of the prime stimuli until the prime-classification task. All things being equal, this new information, along with the change of instructions, should serve to make the primes more visible in the prime-classification task, because subjects are now aware of their presence and can focus solely on them. Nevertheless, and not surprisingly, subjects find the primeclassification task to be very difficult. Thus, it seems reasonable to think that subjects might not be able to sustain the same level of attention and motivation in this task as they did in the experiment proper and, consequently, that the prime-classification task might not be effective in establishing the visibility of the primes.

To guard against this potential "task-difficulty artifact," some researchers have included long-duration primes in the prime-classification task. The reasoning is straightforward. For example, in an earlier study (Finkbeiner, Forster, Nicol, \& Nakamura, 2004), we justified the use of long-duration primes in the prime-classification task by saying that "The purpose of presenting trials with visible primes was to encourage participants to continue trying to do the task. An acceptable accuracy rate on these trials allows us to safely assume that participants were performing the task to the best of their abilities" (p. 12). Several other researchers have included long-duration primes for similar reasons (cf. Grainger, Diependaele, Spinelli, Ferrand, \& Farioli, 2003, p. 1262; Naccache \& Dehaene, 2001). But although some researchers have acted on their intuitions about a task-difficulty artifact by including long-duration primes, Pratte and Rouder (2009) appear to be the first ones to have established empirically the effect of including long-duration primes.

In their Experiment 1, Pratte and Rouder (2009) found that subjects classified short-duration primes $(25 \mathrm{~ms})$ more accurately when long-duration primes $(100 \mathrm{~ms})$ were included in the prime-classification task $(\sim 60 \%)$ than when they were not $(\sim 54 \%)$. This is an important empirical result to have established in the literature. As Pratte and Rouder argued, by increasing the accuracy rate in the primeclassification task (through the inclusion of long-duration primes), the overall task difficulty of the prime-classification task can begin to approximate that of the target-classification task, and this is critical in the endeavor to equate prime processing across the two tasks. Seeing that several researchers were already using Pratte and Rouder's procedure to control for differences in task difficulty, I presume that most researchers will find Pratte and Rouder's argument here to be reasonable.

Having established in Experiment 1 that including longduration primes led to better performance in the primeclassification task for supraliminal primes, Pratte and Rouder then ran two further experiments to determine whether subliminal priming could be observed when controlling for task difficulty. Because their masking procedure had failed to yield chance-level performance in Experiment 1 (even when not controlling for task difficulty), Pratte and Rouder used shorter and shorter prime durations in the next two experiments. Interestingly, they did not observe priming or chance-level performance with $16.7-\mathrm{ms}$ primes (Experiment 2) or 11.8-ms primes (Experiment 3). They did achieve chance-level performance with $8.3-\mathrm{ms}$ primes (Experiment 3), but, not surprisingly, priming did not emerge with this even briefer prime duration.

Pratte and Rouder (2009) concluded from their findings that there is no subliminal priming when the task-difficulty 
artifact is controlled for (p. 1281). They then extended this conclusion to previous studies, suggesting that "previous demonstrations of subliminal priming may simply have reflected a task-difficulty artifact" (p. 1276). But Pratte and Rouder's conclusion that controlling for the task-difficulty artifact eliminates subliminal priming lacks empirical support. Although Pratte and Rouder convincingly showed that supraliminal primes are detected even more accurately when long-duration primes are included in the primeclassification task, it does not follow from this that controlling for task difficulty necessarily affects the classification of subliminal stimuli. Nevertheless, there is no question that the prime- and target-classification tasks differ in overall difficulty and that this difference may influence the extent of prime processing across the two tasks. As for the previous studies that have claimed to provide evidence for subliminal priming while controlling for task difficulty, Pratte and Rouder dismissed these on statistical grounds. Essentially, although these experiments took similar steps to control for task difficulty, they may have failed to reject the null hypothesis (that primes are not visible) due to a lack of power. ${ }^{1}$ The question that remains at this point, then, is whether subliminal priming can be observed in an experiment that (1) controls for the task-difficulty artifact and (2) does not suffer from a lack of power in the prime-classification task. The following experiment was designed to address this question.

\section{The present study}

The purpose of the present experiment was to pursue the possibility of subliminal priming while controlling for task difficulty across the prime- and target-classification tasks. The experiment reported here differs from those of Pratte and Rouder (2009) in several important ways. First, the present experiment employed two prime durations (40 and $50 \mathrm{~ms}$ ) that have produced robust masked congruence priming effects in previous studies (cf. Finkbeiner \& Caramazza, 2008; Finkbeiner, Song, Nakayama, \& Caramazza, 2008). Second, to ensure effective masking with these longer prime durations, I used a masking procedure that is common in the masked priming literature (cf. Grainger et al., 2003; Kouider \& Dupoux, 2004) and that is much stronger than the one used by Pratte and Rouder. Specifically, I used distinct forward and backward masks (see Table 1), with a randomly generated letter string as the backward mask. The importance of using a letter string

\footnotetext{
${ }^{1}$ For a full account of this argument, please see the original article by Pratte and Rouder (2009). See also Rouder, Morey, Speckman, and Pratte (2007), along with Morey, Rouder, and Speckman (2008, 2009).
}

as the backward mask with orthographic primes is in its similarity to the stimulus being masked. ${ }^{2}$ A considerable amount of research has been devoted to establishing the effect of mask-target (or, in this case, "mask-prime") similarity on masking, and the general consensus is that a visually similar (and task-relevant) mask is more powerful than a visually dissimilar one (cf. Enns \& Oriet, 2007; Lleras \& Enns, 2006; Turvey, 1973). Pratte and Rouder used a visually dissimilar and task-irrelevant symbol (“@”) to mask their digit primes.

The third important difference between the present experiment and Pratte and Rouder's (2009) experiments has to do with the prime-classification task. Pratte and Rouder included long-duration primes on $50 \%$ of the trials. One concern with this procedure is that it introduces a new trial type into the prime-classification task that was not present in the target-classification task. Ideally, the only thing that should change between the two tasks is the task instructions. A second concern is that the accuracy rates on the long-duration trials only reflected performance on $50 \%$ of the trials. Perhaps more importantly, the accuracy rates on the long-duration trials $(\sim 80 \%)$ were well below the accuracy rates in the target-classification task ( $95 \%)$. Thus, the inclusion of long-duration primes did not adequately control for the differences in task difficulty.

To address the concerns with Pratte and Rouder's (2009) procedure for controlling task difficulty, the primes in the present experiment were presented in color (red or green) in both the target- and prime-classification tasks. The masks and the targets were presented in white, and all stimuli appeared on a black background. The reason for using colored primes is straightforward: In the prime-classification task, subjects used a single mouse click to indicate the color and identity of the prime stimulus. Though identifying the color of heavily masked prime stimuli requires sharply focused attention, subjects are nevertheless very good at it, and the accuracy rates are approximately equal to those in the target-classification task (both greater than 90\%). Thus, this procedure controls for task difficulty across the two tasks very well. Importantly, subjects are generally unable to identify the stimulus carrying the color, and chance-level performance here allows us to conclude that the critical dimension (i.e., the dimension of the stimulus that produces priming) went undetected.

\footnotetext{
${ }^{2}$ The stimulus to be masked is usually referred to as the target in the masking literature. Here, though, the stimulus to be masked is the prime. As is typical in the masked priming literature, the prime in the present study is backward masked by two successive masks: first by a randomly generated letter string, and then by the target, which is the most powerful backward mask because of its task relevance.
} 
Table 1 Stimuli used in Experiment 1

\begin{tabular}{lll}
\hline Prime Stimuli & Target Stimuli & Masks \\
\hline "dog," "may" & SHEEP, GOAT, KANGAROO, HORSE, PIG & $\begin{array}{l}\text { forward mask: 3\#\&5\%\#\&@92 } \\
\text { backward mask: SWFGHKRPBM }\end{array}$ \\
\hline
\end{tabular}

\section{Method}

The present study is adapted from a version of the masked congruence priming paradigm that has been used previously (cf. Finkbeiner \& Caramazza, 2008). In the specific task used here, subjects indicated whether a target word stimulus was an "animal" word or a "month" word.

\section{Subjects}

Twenty-one undergraduate psychology students participated for course credit.

\section{Stimuli and design}

The stimuli used in the experiment are listed in Table 1. The target stimuli consisted of five animal words and five month words. The prime stimuli were the words "dog" and "may" and were not included in the set of target items. Both the forward and backward masks are also listed in Table 1 . The forward mask always appeared as it is depicted in Table 1; the letters in the backward mask were randomly assigned to different positions on each trial. As mentioned, the masks and targets appeared in white, and the primes appeared in either red or green. All stimuli appeared on a black background. The experiment was controlled by Presentation software (Neurobehavioral Systems) running on PCs with 17 -in. CRT screens $(1,024 \times 768$ resolution, $100-\mathrm{Hz}$ refresh rate).

The trial structure is depicted in Fig. 1. Each trial began with a fixation cross and four white points $\left(0.2^{\circ}\right.$ of visual angle) at the corners of an imaginary square $\left(15^{\circ}\right)$. The four white points appeared statically for $100 \mathrm{~ms}$ and then moved smoothly toward the fixation cross over 90 screen refreshes (900 ms), whereupon the forward mask was presented for $100 \mathrm{~ms}$. The prime was presented for either 40 or $50 \mathrm{~ms}$, followed by the backward mask for 10 or $20 \mathrm{~ms}$ (fixing prime-target SOA at $60 \mathrm{~ms}$ ) and then the target stimulus for $300 \mathrm{~ms}$. Each stimulus followed the preceding one immediately, without any intervening video frames. Subjects initiated a trial by pressing both the "Z" key with their left hand and the " " key with their right hand. When the target appeared, subjects responded by pressing either the "Z" key for animal words or the " " key for month words. Subjects were instructed to respond as quickly and as accurately as possible.

Trials were presented in six blocks of 80 trials each. The sequence of trials was randomly generated at the beginning of each block with two constraints: (1) targets were not allowed to repeat on immediately successive trials, and (2) no more than three responses of one type (e.g., "animal" or "month") were allowed in succession. In each block, each target stimulus $(N=10)$ was rotated through each of the eight design cells that were formed by crossing the factors Prime Color (green and red), Prime Duration (40 and $50 \mathrm{~ms}$ ), and Prime Type (congruent and incongruent). ${ }^{3}$ The first block was a practice block and is not included in the analysis below. The experiment proper consisted of Blocks 2 and 3. The remaining three blocks (240 trials) were assigned to the prime-classification task.

The prime-classification task was identical to the experiment proper in every way, except that after their buttonpress response to the target, subjects were asked to use the computer mouse to indicate the color and identity of the prime stimulus (see Fig. 2). The instructions for the prime-classification task were as follows: "As before, if you see an animal word, press the ' $\mathrm{Z}$ ' on the keyboard. If you see a month word, press the '/' on the keyboard. After you make your response, please use the mouse to click on the distractor." To equate prime processing across the primeand target-classification tasks as closely as possible, primeclassification responses were only elicited on trials with correct target-classification responses.

As is evident in Fig. 2, our procedure allowed us to obtain both a color accuracy score and an identity accuracy score from a single mouse click. To be clear, consider the case in which the prime was the word "dog" in green. If the subject clicked on either the red or the green "dog," he/she was given a correct identity score. If the subject clicked on either of the two green possibilities, he/she was given a correct color score.

\section{Results}

Target classification

Incorrect responses $(3.5 \%)$ were excluded from the RT analyses, as were trials with RTs below $100 \mathrm{~ms}(<0.1 \%)$. A 2 (prime duration: 40 vs. $50 \mathrm{~ms}$ ) $\times 2$ (prime type: congruent vs. incongruent) repeated measures ANOVA was conducted

\footnotetext{
${ }_{3}^{3}$ Prime Color was a dummy factor that was included only to balance the number of red and green primes across the experimental conditions equally.
} 
Fig. 1 Trial structure used in Experiment 1. Each trial began with four dots moving smoothly toward the fixation point, and then the forward mask for $100 \mathrm{~ms}$. The primes were presented for 40 or $50 \mathrm{~ms}$, and the backward mask was presented for 10 or $20 \mathrm{~ms}$ to keep the prime-target SOA constant at $60 \mathrm{~ms}$

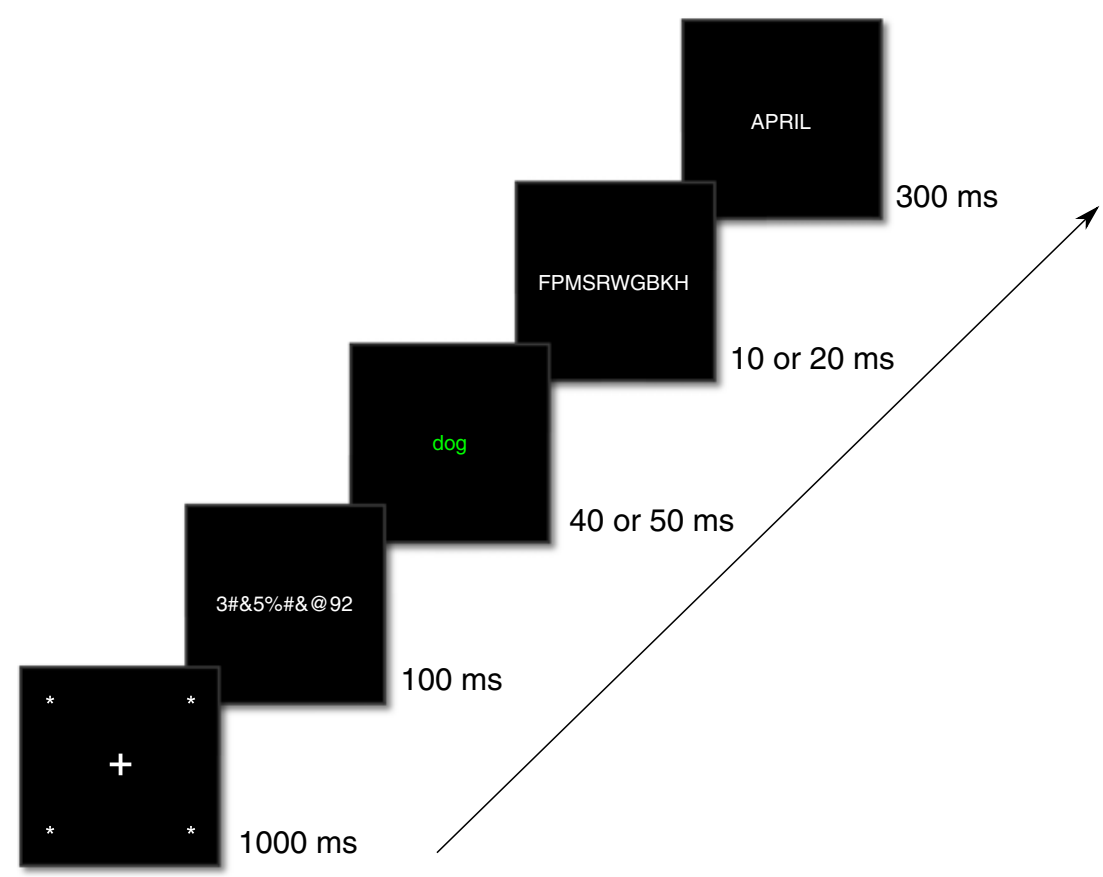

over RTs. This ANOVA revealed a main effect of prime type $[F(1,20)=23.64, p<.01]$ but no effect of prime duration and no interaction between the two factors $\left(F_{\mathrm{S}}<1\right)$. The planned comparisons revealed significant priming effects with both 40-ms primes $[t(20)=2.5, p<.05]$ and 50 -ms primes $[t(20)=2.2, p<.05]$. These results (see Fig. 3a) replicate several findings reported in the literature (cf. Finkbeiner \& Forster, 2008; Kouider \& Dehaene, 2007, for reviews), although this is the first demonstration of masked congruence priming that we are aware of with distinctly colored prime stimuli.

\section{Prime classification}

As is clear in Fig. 3b, color accuracies were very high $(M=92.1 \%)$ and did not differ as a function of prime

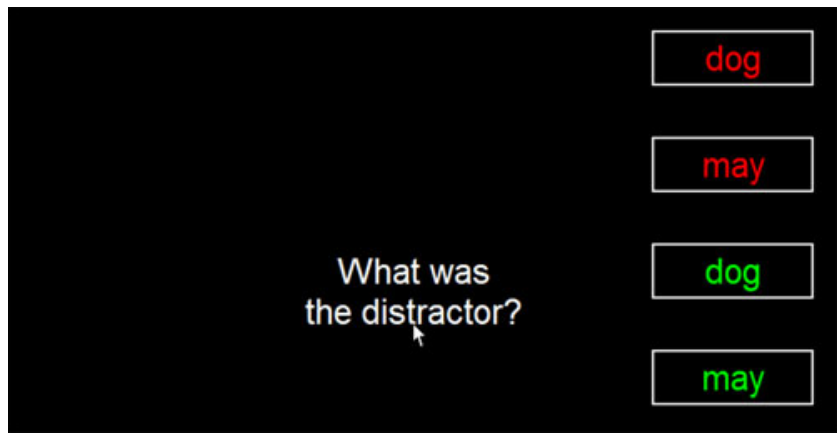

Fig. 2 Prime-classification task used in Experiment 1. Subjects responded with the computer mouse by clicking on the appropriate item on the right. A single mouse click yielded both a color accuracy score and an identity accuracy score. See the text for details duration $(p=.31)$. These high accuracy scores, which approximate the accuracy scores in the target-classification task, indicate near-perfect control of task difficulty.

Of more interest are the prime identification scores. To ensure that the prime identification scores reflected the subjects' best possible performance, only those trials with correct color choices were considered. These results are plotted in Fig. 3b. With 40-ms primes, the mean accuracy rate was $51.8 \%\left(d^{\prime}=0.098\right.$; one-sample $t$ test: $t(20)=1.67$, $p=.19)$, and with $50-\mathrm{ms}$ primes it was $55.6 \%\left(d^{\prime}=0.308\right.$; one-sample $t$ test: $t(20)=2.43, p=.02)$. Identification scores did not vary with prime color for the 40-ms primes (red primes, 50.97\%; green primes, $51.92 \%$ ) or the $50-\mathrm{ms}$ primes (red primes, 54.78\%; green primes, 56.28\%), with all $t$ values less than 1 . To establish whether primeclassification accuracy rates differed from chance, we first used a traditional one-sample $t$ test. With the 40-ms primes (see Table 2 for response frequencies by prime item), the $t$ test indicated a nonsignificant difference from chance, $t(20)=1.56, p=.1347$; with the $50-\mathrm{ms}$ primes, there was a significant difference from chance, $t(20)=2.45, p=.02$.

At first blush, these results suggest that we have observed subliminal priming with the 40-ms primes. But the present study, despite including 1.5 times as many trials in the prime-classification task $(N=240)$ as the targetclassification task $(N=160)$, is still susceptible to the criticism that Rouder, Morey, and colleagues have persuasively leveled against many subliminal priming studies (cf. Morey, Rouder, \& Speckman, 2008, 2009; Rouder, Morey, Speckman, \& Pratte, 2007). Namely, the present design, with 21 subjects and 240 prime-classification trials, still 

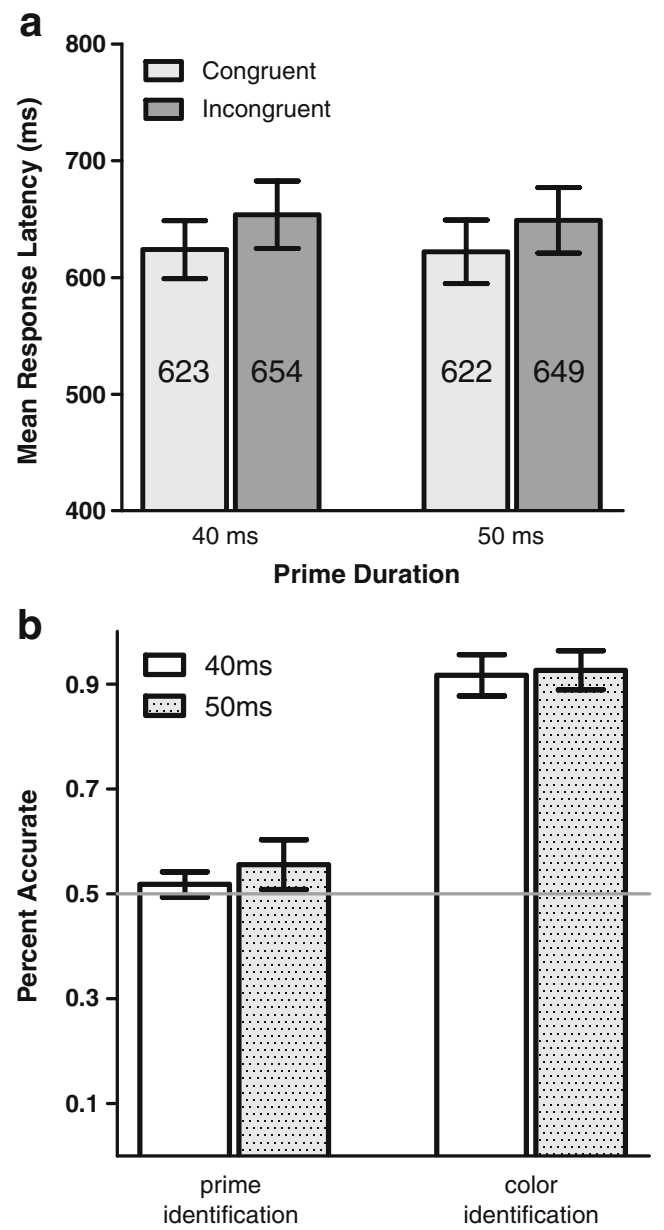

Fig. 3 Mean response latencies by condition (panel A) and mean accuracy rates in the prime-classification task (panel B) by prime duration and stimulus dimension (color or identity). Error bars represent $95 \%$ confidence intervals

lacks sufficient power for the standard significance test approach to accurately resolve performance that is only slightly above chance levels. It may seem that the solution to this problem would be to increase the power of the experiment. But this approach is ultimately not feasible when working with human subjects. As the subjects' true performance comes to approximate chance performance more and more closely, the number of trials needed for the $t$ test approach increases beyond the reach of most experimental designs. For example, with a sample size of 21 and a mean true performance of $52 \%$, one would need approximately 570 prime-classification trials to bring the probability of wrongly accepting the null hypothesis down

Table 2 Response frequencies by prime item with 40 -ms primes below $5 \%$. With a true performance of $51 \%$, approximately 2,300 trials would be needed. This is the null-sensitivity problem, and trying to resolve it through the addition of more and more trials is not practical for most researchers, not to mention for their subjects. For this reason, among others, Merikle and Reingold have argued against using standard null-hypothesis significance testing (NHST) in the study of subliminal perception (Merikle \& Reingold, 1998; Reingold \& Merikle, 1988)

Fortunately, the standard NHST approach is not the only way to test for chance-level performance. In a series of recent articles, Rouder, Morey, and colleagues (Morey et al., 2008, 2009; Rouder et al., 2007) have introduced a hierarchical model within the Bayesian framework that offers researchers a way to resolve the null-sensitivity problem. They have termed this the mass-at-chance model (MAC). The MAC model assumes that each subject's performance reflects a true latent ability score that, like a $z$ score, varies both positively and negatively. Latent abilities below zero correspond to at-chance true performance; latent abilities above zero correspond to above-chance performance. Across a population of subjects, these true latent abilities are assumed to be normally distributed. With this assumption, it is possible to calculate the posterior probability that each individual has a latent ability that is below zero - that is, the probability that an individual has true subliminal performance. If this posterior probability is above some criterion, say .95, then that individual is judged to be performing at chance. A virtue of MAC is that it penalizes underpowered designs, thereby mitigating the null-sensitivity problem. Small sample sizes yield highly variable posterior estimates of subjects' latent abilities, which makes it more difficult to claim subliminality (Rouder et al., 2007).

The prime-classification data from the present experiment were analyzed using the one-parameter MAC model (Morey et al., 2008), which is available as a package for the $R$ language (http://r-forge.r-project.org/projects/massatchance/). The results of this analysis are presented in Fig. 4, where the posterior probabilities for each individual are plotted as a function of accuracy. The circles indicate subjects' performance with 40-ms primes, and the squares indicate performance with 50-ms primes. The dashed line indicates the criterion of .95, and symbols above this line are filled. As is clear, subliminality was confirmed for only 1 subject in the condition with 50 -ms primes. In contrast, 16 of the 21 subjects were judged to be at chance with the 40-ms primes. Following Rouder et al. (2007), the RTs for the 17 subjectby-prime-duration combinations for which subliminality was confirmed were entered into a paired-samples $t$ test (twotailed) to determine whether subliminal priming had occurred. The results of this $t$ test indicated a significant subliminal priming effect $[t(16)=2.79, p=.01]$. To quantify 


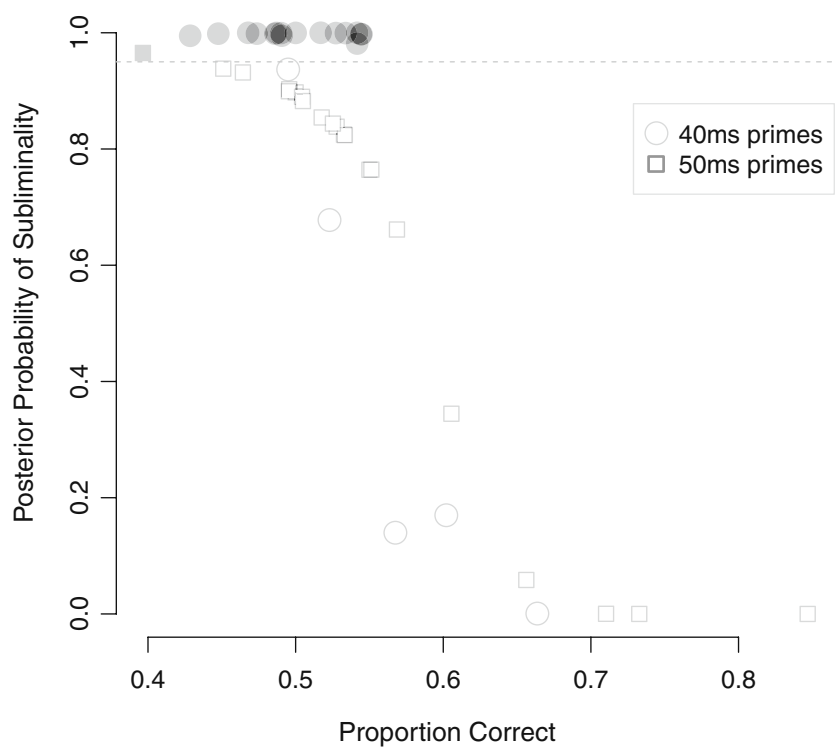

Fig. 4 Estimated posterior probabilities from the one-parameter MAC model for each individual subject as a function of prime duration and accuracy in the prime-classification task. The circles indicate subjects' performance with $40-\mathrm{ms}$ primes, and the squares indicate performance with $50-\mathrm{ms}$ primes. The dashed line indicates the criterion of .95 . Filled symbols indicate individuals for whom subliminality was confirmed by the MAC model

the evidence in favor of the alternative hypothesis, we used the Bayes factor calculator (Rouder, Speckman, Sun, Morey, $\&$ Iverson, 2009). In this case, the sample size of 17 and a $t$ value of 2.79 yield a Bayes factor of 0.26 . This means that the alternative hypothesis (i.e., that prime type modulates RTs) is 3.85 times $(1 / .26)$ more likely than the null hypothesis (i.e., that prime type does not modulate RTs).

It should be noted that the criterion of .95 is very strict, and may be too strict for this application (Jeff Rouder, personal communication). If one were to relax the criterion of subliminality to .90 , which corresponds to 1 -in-9 odds, then the MAC analysis would indicate 22 subject-by-primeduration combinations for which subliminality was confirmed (see Fig. 5). A $t$ test over these 22 data points again revealed a reliable effect of priming $[t(21)=3.47, p<.01]$, with a Bayes factor of 0.06. Thus, with 9:1 odds in favor of subliminality, this experiment yielded 16:1 odds in favor of priming. Perhaps more important, though, is the overall relationship between priming and the estimate of subliminality, which is depicted in Fig. 5. The gray vertical line corresponds to a subliminality criterion of .90 . The circles (40-ms primes) and squares (50-ms primes) indicate the magnitude of priming as a function of the estimate of subliminality. As is clear from the fitted polynomial (LOESS), the magnitude of the priming effect does not decrease as the criterion for subliminality increases. Thus, the evidence for subliminal priming in the present exper- iment does not appear to depend upon a generous or overly relaxed criterion of subliminality.

\section{Discussion}

The motivation for the present study was twofold. First, we set out to develop a prime-classification procedure that guarded against the task-difficulty artifact highlighted by Pratte and Rouder (2009). Our solution was to present the prime stimuli in red or green in both the experiment proper and the prime-classification task. In the prime-classification task, subjects reported both the color and identity of the primes. The findings indicate that our procedure was effective in guarding against the task-difficulty artifact, since subjects were highly successful in reporting the prime's color $(M=92.1 \%)$. This near-ceiling level of performance approximates the accuracy levels in the targetclassification task, indicating almost perfect control for task difficulty. Nevertheless, subjects' performance on the critical dimension of the stimulus - the word's identity - did not differ from chance with the briefer of the two prime durations. Critically, a priming effect was observed (16:1 odds) for the subject-by-prime-duration combinations for which the MAC analysis confirmed subliminality ( $9: 1$ odds) in the primeclassification task. Thus, the conclusion of this study stands in

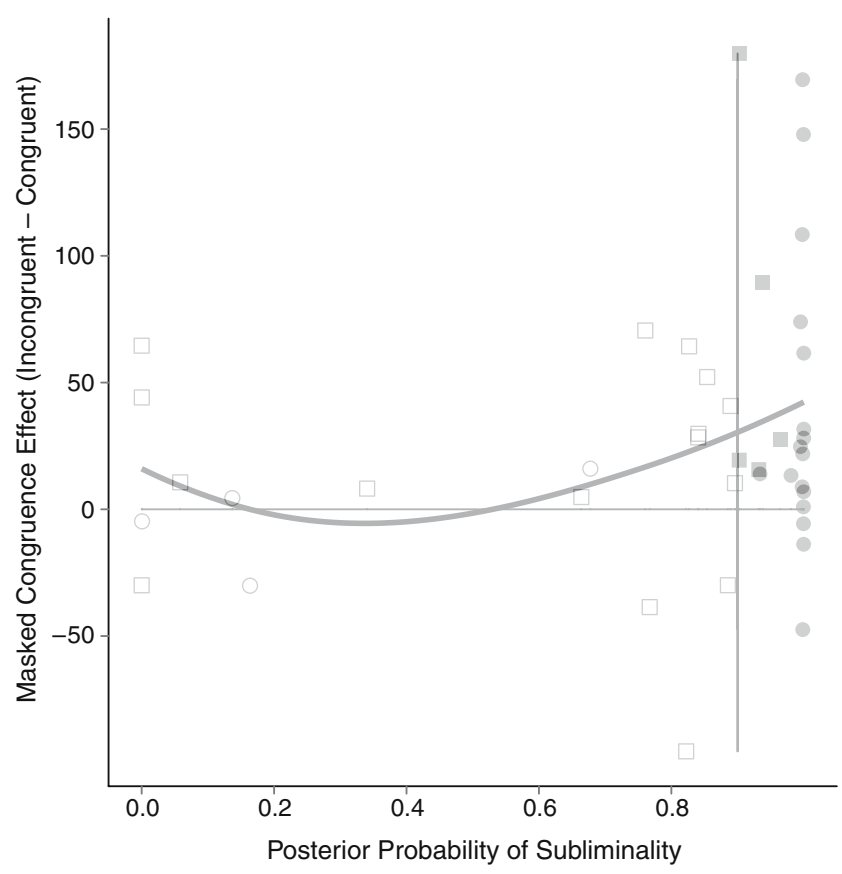

Fig. 5 Masked congruence effect in milliseconds for 40-ms primes (circles) and 50-ms primes (squares) as a function of the estimated posterior probability of subliminality. Filled symbols correspond to subject-by-prime-duration combinations that exceed the subliminality criterion of .90. The fitted polynomial (LOESS) suggests that priming does not decrease as the probability of subliminality increases 
sharp contrast to that of Pratte and Rouder: Namely, I conclude that controlling for task difficulty does not eliminate subliminal priming.

How did the two studies arrive at such different conclusions? There are at least three possible reasons, which I consider here. The first has to do with how the studies pursued chance-level performance. Pratte and Rouder (2009) chose to manipulate prime duration, instead of the strength of masking, in their pursuit of chance performance. This decision led them to use increasingly brief prime durations to achieve subliminality, which they finally did with 8.3 -ms primes. However, because they failed to observe priming with supraliminal primes at slightly longer durations (e.g., $11.8 \mathrm{~ms}$ ), it is not surprising that they also failed to observe priming with the even briefer $8.3-\mathrm{ms}$ primes. I chose a different approach in the present study. Specifically, I began with prime durations (40 and $50 \mathrm{~ms}$ ) that are known to produce priming and then used a masking procedure much stronger than the one used by Pratte and Rouder to achieve chance-level performance in the prime-classification task.

A general rule of thumb when using pattern masking is that "like masks like." Or, as Turvey (1973) put it, "the more similarities between the target and mask ... the greater the opportunity for masking" (p. 47). Similarly, Enns (2004) compared several different backward masks for letter targets and found that when the mask followed shortly after the target, a letter mask was much better than a digit, which was much better than a noise pattern of random dots. In the present study, I followed this rule of thumb by using a letter string to backward mask the prime, which itself was a letter string. Pratte and Rouder (2009), on the other hand, used a nondigit mask (“@”) to mask digit primes. This decision, along with their use of identical forward and backward masks, which causes the prime to "pop out" or appear in relief, contributed to the lack of effective masking in their experiment.

A second possible reason for Pratte and Rouder's (2009) failure to observe subliminal priming in the experiments that produced supraliminal priming (e.g., Experiment 1) has to do with the overlap between the target-induced responseslearned during the course of the experiment - and the primeinduced responses in the prime-classification task. In Pratte and Rouder's experiments, the prime and target stimuli were drawn from the same stimulus set and, unless the appropriate precautionary steps are taken, this can be problematic for the prime-classification measure. In his response to Dehaene et al. (1998), Damian (2001) demonstrated very clearly that when a small number of targets are used, subjects quickly learn the appropriate association between each stimulus and its corresponding response and that they can then perform the task using these S-R mappings. Furthermore, he observed that these learned S-R mappings could be "triggered" automatically by a masked (subliminal) prime stimulus. Damian was concerned with the question of whether masked congruence priming effects should be attributed to a semantic or a nonsemantic source, but his findings are relevant to the prime-classification task, too. If a nonconsciously perceived stimulus can trigger a motor response, and we think that this is a robust phenomenon (cf. Finkbeiner \& Friedman, in press; Finkbeiner et al., 2008), then above-chance performance in the prime-classification task may index nonconsciously elicited motor processesnot visual awareness. Most researchers are well aware of this issue and guard against it by requiring subjects to wait for a brief period of time (e.g., $1 \mathrm{~s}$ ) before responding in the prime-classification task (cf. Finkbeiner \& Caramazza, 2008; Vorberg, Mattler, Heinecke, Schmidt, \& Schwarzbach, 2003). The idea here is that after a short period of time the prime-induced response will have decayed sufficiently so that the subject's prime-classification response will reflect the contents of their visual awareness as opposed to nonconsciously elicited motor responses. Nevertheless, it appears that Pratte and Rouder let their subjects respond with the same keypresses and with the same rapidity in both the prime- and target-classification tasks. In the present study, two precautionary steps were taken to prevent nonconsciously induced motor responses from contaminating the prime-classification measure. First, the prime and target stimuli were drawn from distinct sets, thereby mitigating the influence that learned $\mathrm{S}-\mathrm{R}$ mappings might have on the prime-classification measure. Second, prime and target classifications required separate motor responses. In the experiment proper, subjects responded with a buttonpress, whereas in the prime-classification task, they responded with a mouse click.

The third possibility, which is related to the second, is that the prime-classification measure in the present experiment may not have been sufficiently sensitive. That is, although Pratte and Rouder (2009) may not have been careful enough to prevent nonconsciously triggered responses from contaminating their prime-classification measure, perhaps the present study went too far in the other direction. In particular, there are two aspects of the prime-classification task used in the present experiment that might be of concern. The first of these has to do with the combination of color and identity into a single response in the prime-classification task. Namely, in the targetclassification task, subjects only attended to whether the target was a month word or an animal word, but in the prime-classification task, they were asked to report both the color and identity of the prime. The concern here is that, because color is the easier of the two dimensions to report, subjects might have focused solely on the color of the stimulus and ignored its identity. While at first glance this seems like a plausible strategy that subjects might adopt, 
there are two reasons to think that they did not. The first has to do with the simple fact that subjects may not have been able to adopt this strategy despite their best efforts. By way of explication, try, for example, to look directly at an object on your desk or on this page and observe its color but not its shape. This presumably will be quite difficult. Humans generally cannot see the color of a temporally and spatially attended object at fixation without seeing the object itselfunless perception of the object's form has been uniquely disrupted through masking. The second reason to think that subjects did not focus solely on the color of the primes comes from the accuracy rates across the two prime durations. If the prime-classification task led subjects to focus solely on the prime's color, the accuracy rates would not have differed as a function of prime duration. Yet both the standard analysis and the MAC analysis revealed that subjects were much better at identifying the 50-ms primes than the 40-ms primes. This suggests that the primeclassification task in the present study was sufficiently sensitive to be diagnostic of our subjects' ability to identify the prime stimuli.

The second aspect of the prime-classification task that may be of concern is that subjects were required to make two responses: first to the target stimulus, and then to the prime. The reason for this was to equate the two tasks as closely as possible. As mentioned above, the primeclassification task is only valid if the primes are equally visible in the target- and prime-classification tasks (cf. Reingold \& Merikle, 1988). One way to equate the extent of prime processing in the two tasks is to have subjects perform the prime-classification task in the context of the target-classification task, but a reasonable concern is that the primes may have been available for report immediately upon presentation but were then lost while responding to the target. Again, there are two reasons to think that this is unlikely.

The first comes from the results of a previous study that used a within-subjects design to compare these two different ways of conducting the prime-classification task. In that study (Finkbeiner \& Caramazza, 2008, Experiment 5), individuals participated in five experimental sessions, two of which involved target classification and three of which involved prime classification (one session per day). The experiment tested for priming and prime visibility across six prime durations $(10-60 \mathrm{~ms}$, in 10 -ms increments); each session included 600 trials (100 per prime duration). Two of the prime-classification sessions asked subjects to first respond to the target and then to the prime. In the third prime-classification session, subjects responded to the prime only, ignoring the target. Critically for our purposes here, the measure of prime awareness $\left(d^{\prime}\right)$ revealed the same pattern across both tasks, in which the shortest three prime durations were deemed subliminal and the longest three durations were deemed supraliminal. That is, the subjects' ability to report the primes was (statistically) the same, regardless of whether or not they responded to the target stimulus first. ${ }^{4}$

The second reason to doubt that having subjects respond to the target impaired their ability to report the prime comes from the results of the present study. Remember that the concern here is that the primes were clearly visible to the subjects initially but were subsequently forgotten while responding to the target stimulus. To accept this possibility, one would have to argue that subjects were better at identifying the 50-ms primes because they had sufficient time with those primes (but not with the 40-ms primes) to encode and begin rehearsing the prime-related information. Or, to put it differently, one would have to argue that our subjects were unable to encode a 40-ms stimulus into memory, despite its being briefly visible to the subject. But we know that this was not true by virtue of our subjects being able to report the color of the 40 -ms primes (>90\%) after they responded to the target. Thus, it seems more likely that the difference between the 40- and 50-ms primes was the visibility of the prime's identity. Along these lines, it bears mentioning that a hallmark of conscious percepts is their stability and, thus, their availability for other cognitive operations and tasks (Dehaene, Changeux, Naccache, Sackur, \& Sergent, 2006; Dehaene \& Naccache, 2001). In summary, it seems reasonable to think that the prime-classification measure used in the present study was sufficiently sensitive to reveal above-chance performance by virtue of its success in revealing ceiling-level performance with both 40- and 50ms primes along one dimension (color) and in revealing above-chance performance with the 50 -ms primes along the other dimension (identity).

\section{Conclusion}

Demonstrating subliminal priming is difficult to do for multiple reasons, and claims of subliminal priming are routinely challenged. The most recent challenge has come from Pratte and Rouder (2009), who highlighted a methodological artifact in the prime-classification task that, they contended, has led researchers to wrongly claim evidence for subliminal priming. Essentially, the primeclassification task is, by design, much more difficult for subjects than the target-classification task in the experiment proper. And, as Pratte and Rouder argued, unless one

\footnotetext{
${ }^{4}$ It should be noted that this experiment revealed reliable priming effects despite controlling for task difficulty by including visible longduration primes. The primes that were presented for 40 to $\sim 60 \mathrm{~ms}$ were visible $\left(d^{\prime}>2.0\right)$; the primes presented for less than $40 \mathrm{~ms}$ were not visible ( $d^{\prime}$ not different from zero), and yet they produced reliable priming effects.
} 
controls the difficulty of the two tasks, the subjects' poor performance in the prime classification task might reflect the overall difficulty of the task, as opposed to the subliminality of the primes.

In the present study, we controlled the difficulty of the two tasks by presenting the primes in color and by having subjects report both the color and the identity of the primes. Subjects reported the color of the primes very accurately (>90\%), approximating the accuracy levels in the experiment proper. Thus, we were successful in controlling the difficulty of the two tasks. Despite our having controlled the task difficulty, subjects were not able to identify the primes at better-than-chance levels when the primes were presented for $40 \mathrm{~ms}$, but they could when primes were presented for $50 \mathrm{~ms}$. Thus, we were successful in masking the $40-\mathrm{ms}$ primes. Critically, we observed significant priming effects with the 40ms primes, even when we restricted our analysis to just those subjects for whom subliminality had been confirmed by the mass-at-chance model (Morey et al., 2008, 2009; Rouder et al., 2007). Thus, we conclude that subliminal priming effects can be observed even when taking the steps necessary to guard against the task-difficulty artifact. We suggest that our findings stand in contrast to those of Pratte and Rouder (2009) because of important differences in the masking procedures between the two studies.

Author Note I am grateful to Jeff Rouder for his comments and suggestions on an earlier draft of the manuscript; to Richard Morey for making the one-parameter MAC model available as an R package; and to Shahd Al-Janabi for collecting the data. This work was supported in part by an Australian Research Fellowship from the Australian Research Council (DP0880806).

\section{References}

Abrams, R. L., Klinger, M. R., \& Greenwald, A. G. (2002). Subliminal words activate semantic categories (not automated motor responses). Psychonomic Bulletin \& Review, 9, 100-106.

Damian, M. F. (2001). Congruity effects evoked by subliminally presented primes: Automaticity rather than semantic processing. Journal of Experimental Psychology: Human Perception and Performance, 27, 154-165.

de Gardelle, V., \& Kouider, S. (2010). How spatial frequencies and visual awareness interact during face processing. Psychological Science, 21, 58-66.

Dehaene, S., Changeux, J. P., Naccache, L., Sackur, J., \& Sergent, C. (2006). Conscious, preconscious, and subliminal processing: A testable taxonomy. Trends in Cognitive Sciences, 10, 204-211.

Dehaene, S., \& Naccache, L. (2001). Towards a cognitive neuroscience of consciousness: Basic evidence and a workspace framework. Cognition, 79, 1-37.

Dehaene, S., Naccache, L., Le Clec'H, G., Koechlin, E., Mueller, M., Dehaene-Lambertz, G., et al. (1998). Imaging unconscious semantic priming. Nature, 395, 597-600.

Eimer, M., \& Schlaghecken, F. (1998). Effects of masked stimuli on motor activation: Behavioral and electrophysiological evidence.
Journal of Experimental Psychology: Human Perception and Performance, 24, 1737-1747.

Enns, J. T. (2004). Object substitution and its relation to other forms of visual masking. Vision Research, 44, 1321-1331.

Enns, J. T., \& Oriet, C. (2007). Visual similarity in masking and priming: The critical role of task relevance. Advances in Cognitive Psychology, 3, 211-226.

Finkbeiner, M., \& Caramazza, A. (2008). Modulating the masked congruence priming effect with the hands and the mouth. Journal of Experimental Psychology: Human Perception and Performance, 34, 894-918.

Finkbeiner, M., \& Forster, K. I. (2008). Attention, intention and domainspecific processing. Trends in Cognitive Sciences, 12, 59-64.

Finkbeiner, M., \& Friedman, J. (in press). The flexibility of nonconsciously deployed cognitive processes: Evidence from masked congruence priming, PLOS ONE.

Finkbeiner, M., Forster, K. I., Nicol, J., \& Nakamura, K. (2004). The role of polysemy in masked semantic and translation priming. Journal of Memory and Language, 51, 1-22.

Finkbeiner, M., \& Palermo, R. (2009). The role of spatial attention in nonconscious processing: A comparison of face and nonface stimuli. Psychological Science, 20, 42-51.

Finkbeiner, M., Song, J. H., Nakayama, K., \& Caramazza, A. (2008). Engaging the motor system with masked orthographic primes: A kinematic analysis. Visual Cognition, 16, 11-22.

Forster, K. I., \& Davis, C. (1984). Repetition priming and frequency attenuation in lexical access. Journal of Experimental Psychology: Learning, Memory, and Cognition, 10, 680-698.

Grainger, J., Colé, P., \& Segui, J. (1991). Masked morphological priming in visual word recognition. Journal of Memory and Language, 30, 370-384.

Grainger, J., Diependaele, K., Spinelli, E., Ferrand, L., \& Farioli, F. (2003). Masked repetition and phonological priming within and across modalities. Journal of Experimental Psychology: Learning, Memory, and Cognition, 29, 1256-1269.

Kiefer, M., \& Brendel, D. (2006). Attentional modulation of unconscious "automatic" processes: Evidence from eventrelated potentials in a masked priming paradigm. Journal of Cognitive Neuroscience, 18, 184-198.

Koechlin, E., Naccache, L., Block, E., \& Dehaene, S. (1999). Primed numbers: Exploring the modularity of numerical representations with masked and unmasked semantic priming. Journal of Experimental Psychology: Human Perception and Performance, 25, 1882-1905.

Kouider, S., \& Dehaene, S. (2007). Levels of processing during nonconscious perception: A critical review of visual masking. Philosophical Transactions of the Royal Society B, 362, 857-875.

Kouider, S., \& Dehaene, S. (2009). Subliminal number priming within and across the visual and auditory modalities. Experimental Psychology, 56, 418-433.

Kouider, S., \& Dupoux, E. (2004). Partial awareness creates the "illusion" of subliminal semantic priming. Psychological Science, $15,75-81$.

Kouider, S., Eger, E., Dolan, R., \& Henson, R. N. (2009). Activity in face-responsive brain regions is modulated by invisible, attended faces: Evidence from masked priming. Cerebral Cortex, 19, 13-23.

Kunde, W., Kiesel, A., \& Hoffmann, J. (2003). Conscious control over the content of unconscious cognition. Cognition, 88, 223-242.

Lleras, A., \& Enns, J. T. (2006). How much like a target can a mask be? Geometric, spatial, and temporal similarity in priming: A reply to Schlaghecken and Eimer (2006). Journal of Experimental Psychology: General, 135, 495-500.

Merikle, P. M., \& Reingold, E. M. (1998). On demonstrating unconscious perception: Comment on Draine and Greenwald (1998). Journal of Experimental Psychology: General, 127, 304-310. 
Morey, R. D., Rouder, J. N., \& Speckman, P. L. (2008). A statistical model for discriminating between subliminal and near-liminal performance. Journal of Mathematical Psychology, 52, 21-36.

Morey, R. D., Rouder, J. N., \& Speckman, P. L. (2009). A truncatedprobit item response model for estimating psychophysical thresholds. Psychometrika, 74, 603-618.

Naccache, L., \& Dehaene, S. (2001). Unconscious semantic priming extends to novel unseen stimuli. Cognition, 80, 215-229.

Pessiglione, M., Schmidt, L., Draganski, B., Kalisch, R., Lau, H., Dolan, R. J., et al. (2007). How the brain translates money into force: A neuroimaging study of subliminal motivation. Science, 316, 904-906.

Pratte, M. S., \& Rouder, J. N. (2009). A task-difficulty artifact in subliminal priming. Attention, Perception, \& Psychophysics, 71, 1276-1283.

Reingold, E. M., \& Merikle, P. M. (1988). Using direct and indirect measures to study perception without awareness. Perception \& Psychophysics, 44, 563-575.

Reynvoet, B., Gevers, W., \& Caessens, B. (2005). Unconscious primes activate motor codes through semantics. Journal of Experimental Psychology: Learning, Memory, and Cognition, 31, 991-1000.

Rouder, J. N., Morey, R. D., Speckman, P. L., \& Pratte, M. S. (2007). Detecting chance: A solution to the null sensitivity problem in subliminal priming. Psychonomic Bulletin \& Review, 14, 597605.

Rouder, J. N., Speckman, P. L., Sun, D. C., Morey, R. D., \& Iverson, G. (2009). Bayesian $t$ tests for accepting and rejecting the null hypothesis. Psychonomic Bulletin \& Review, 16, 225-237.

Turvey, M. T. (1973). On peripheral and central processes in vision: Inferences from an information-processing analysis of masking with patterned stimuli. Psychological Review, 80, 1-52.

Van den Bussche, E., Segers, G., \& Reynvoet, B. (2008). Conscious and unconscious proportion effects in masked priming. Consciousness and Cognition, 17, 1345-1358.

Van Opstal, F., Reynvoet, B., \& Verguts, T. (2005). How to trigger elaborate processing? A comment on Kunde, Kiesel, and Hoffmann (2003). Cognition, 97, 89-97.

Verleger, R., Kötter, T., Jaśkowski, P., Sprenger, A., \& Siebner, H. (2006). A TMS study on non-consciously triggered response tendencies in the motor cortex. Experimental Brain Research, 173, 115-129.

Vorberg, D., Mattler, U., Heinecke, A., Schmidt, T., \& Schwarzbach, J. (2003). Different time courses for visual perception and action priming. Proceedings of the National Academy of Sciences, 100, $6275-6280$. 